Document downloaded from:

http://hdl.handle.net/10251/56195

This paper must be cited as:

Camba, JD.; Contero, M. (2015). Assessing the impact of geometric design intent annotations on parametric model alteration activities. Computers in Industry. 71:35-45. doi:10.1016/j.compind.2015.03.006.

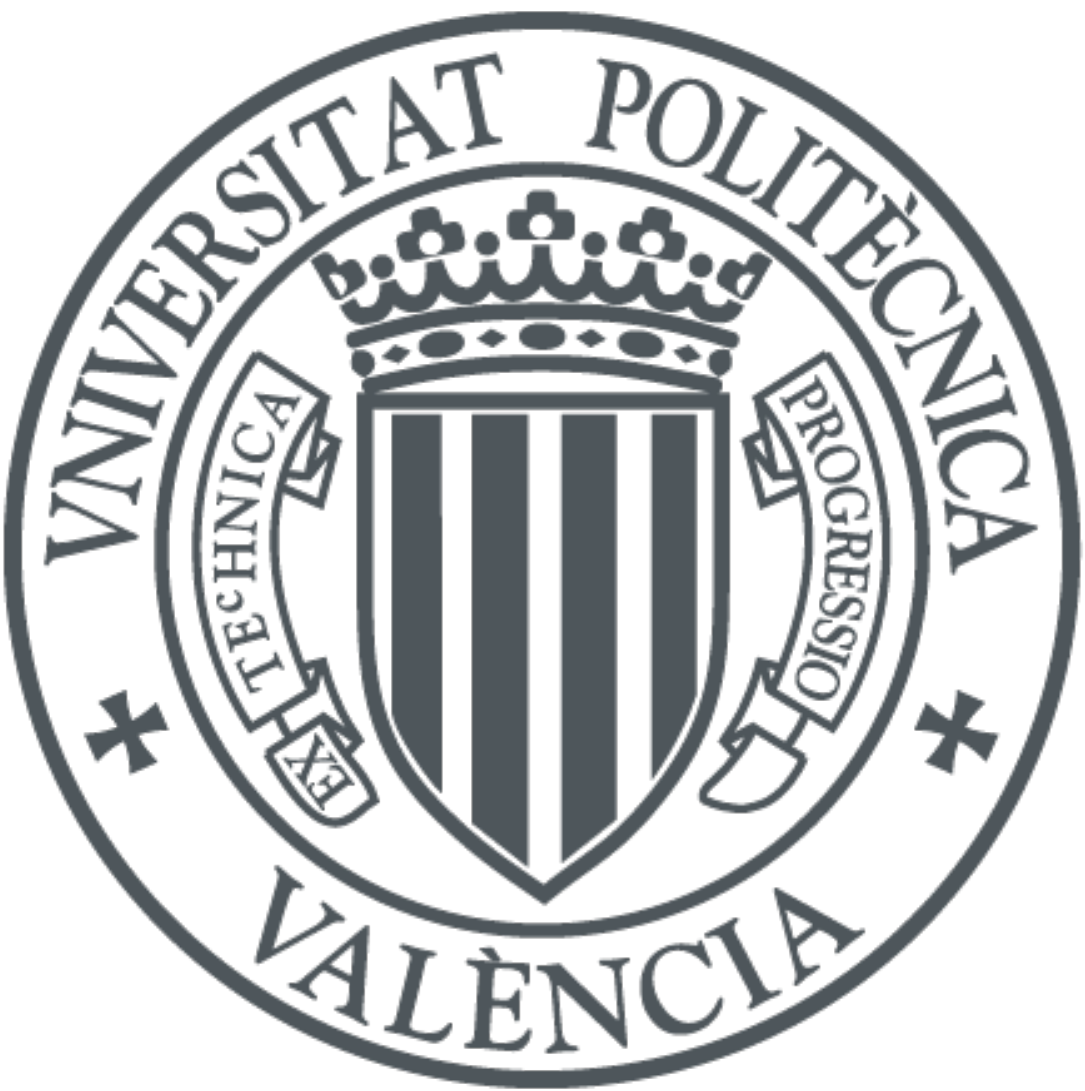

The final publication is available at

http://dx.doi.org/10.1016/j.compind.2015.03.006

Copyright Elsevier

Additional Information 


\title{
Assessing the Impact of Geometric Design Intent Annotations on Parametric Model Alteration Activities
}

\author{
Jorge D. Camba \\ Dwight Look College of Engineering, Texas A\&M University, College Station, TX, United States \\ Manuel Contero \\ Universitat Politècnica de València, I3BH, Valencia, Spain
}

\begin{abstract}
The effective representation and communication of design intent plays a crucial role in CAD model alteration activities. In historybased parametric modeling systems, design intent information is usually expressed implicitly within the model. However, there is evidence that suggests that an explicit representation can increase productivity and quality and facilitate the transferring of design knowledge throughout the different stages of the product lifecycle. In this paper, we assess the effectiveness of $3 \mathrm{D}$ annotations as mechanisms for explicit design intent representation and examine their impact in model alteration processes that require a direct interaction with the model's geometry. We present the results of a series of studies aimed at measuring user performance and model quality in two scenarios. First, we hypothesized that annotations are valuable tools to provide design information when inadequate modeling assumptions can be made by designers. Second, we evaluated annotations as tools to communicate design decisions when multiple options are available. In both cases, results show statistically significant benefits of annotated models, suggesting the use of this technique as a valuable approach to improve design intent communication.
\end{abstract}

Keywords: 3D annotations, CAD model reusability, design intent communication, design reusability, collaborative design.

\section{INTRODUCTION}

In our modern globalized world, the development of new products is a fundamental activity for enterprise survival and competitiveness. Particularly, the transition towards a Model-Based Enterprise (MBE) represents an opportunity for increased performance and efficiency $[1,2]$. Current methods for new product development are largely based on the collaborative engineering paradigm, which relies strongly on the digital representation of the product, usually in the form of CAD models. In fact, it is the efficient use of these digital models what determines the benefits of a successful implementation.

A key factor in new product development activities is the ability to reuse and apply knowledge and designs obtained from previous processes [3]. The high pressure put on engineering firms for delivering better products faster and more economically is giving design reuse (particularly CAD model reuse) an essential role to achieve these objectives. In theory, modern CAD systems allow reusability of existing CAD elements, both as templates for new versions and configurations of the design and as a starting point for new product developments. However, the degree of reusability of a CAD model strongly depends on the modeling methodology and the proper definition and communication of the geometric design intent rather than the technology [4].

In feature-based CAD packages, design intent is typically conveyed implicitly within the CAD model in the form of parent-child relations between features of the model, which are typically displayed as a design tree or history tree in the interface of the parametric modeling software. Before the model can be altered, however, the designer must examine the model structure carefully to gain a thorough understanding of the modeling strategy and procedures used, which often requires a significant effort, even for simple alterations. This situation is particularly noticeable when the designer in charge of altering the model is not its original creator [5]. In order to overcome some of the obstacles of the internal representation of design intent, some authors have proposed the use of $3 \mathrm{D}$ annotations as a mechanism to embed this information into the CAD model's geometry, thus making it explicitly available $[6,7,8]$.

In this paper, we present the results of a series of experiments that evaluate the effectiveness of 3D annotation techniques in terms of design intent communication and reusability. We begin by reviewing relevant work in the areas of CAD model reusability (and how it relates to model alteration), design intent communication, and 3D annotations in engineering design. Next, we describe the experimental setup and procedures, and define how annotations were used in the studies. For each experiment, we present a statistical analysis, which confirms the value of annotations as mechanisms to carry design intent information. Finally, we discuss our results and conclude with suggestions of future research lines. 


\section{RELATED WORK}

\subsection{CAD model Reusability}

Engineering design firms are under constant pressure to increase performance, product quality, and innovate while reducing development times and costs. To guarantee competitiveness in modern markets, new methods and processes need to be put into practice [2]. For example, collaborative design methodologies are rapidly replacing traditional sequential product development models, and the Model-Based Enterprise (MBE) paradigm is increasingly gaining popularity.

Modern collaborative design is typically accomplished by different individuals from various disciplines who work independently yet in combination with others in a network of design, modeling, and engineering efforts. In order to ensure that all pieces can fit and work together seamlessly in a final functional product, significant coordination and organizational efforts are required at all levels of the product life cycle, from the big-picture designs to the smallest details, which all demand time and resources. A highly effective approach to collaborative design relies on an efficient leveraging of 3D digital data. In this context, CAD models have become fundamental assets that can be shared among different stakeholders and moved throughout the different stages of the product lifecycle $[9,10,11]$.

A critical factor for a successful implementation of collaborative product design methodologies in industrial environments is the ability to redesign existing products and apply knowledge from previous processes to new design challenges $[3,10,12]$. The importance of design reuse is particularly noticeable in the area of CAD modeling and representation of digital elements. According to the companies that participated in the study conducted by the Aberdeen Group [10], reusing design elements translates into significant savings. This study also reported that the top performing companies intentionally invest time and resources to capitalize on reusability. In the area of automotive engineering, for example, Bodein et al. [13] concluded that reusability of existing CAD models largely determines the modeling strategies followed by designers when creating new products.

There are, however, problems that need to be solved. The main obstacles that impede a practical and effective implementation of CAD model reuse (and thus design reuse) as well as general procedures that companies are putting into practice to overcome them were identified by [10] and are listed in Table 1.

Table 1. Challenges and responses to CAD model reusability (adapted from [10])

\begin{tabular}{|l|l|}
\hline CHALLENGE & PROCEDURE \\
\hline 1. Model modification requires expert CAD knowledge & Train users to increase CAD skills \\
\hline 2. Models are inflexible and fail after changes & Design for wide range of modifications \\
\hline 3. Users cannot find models to reuse & Centralize design data in library accessible structure \\
\hline 4. Only original designer can change models successfully & Detail design information in model \\
\hline
\end{tabular}

There is clear relationship between challenges 1 and 2, as they both involve the creation of better designed models. CAD users need to be trained in CAD methods, tools, and technology, and develop good modeling skills to create models that are reusable and easy to maintain. Challenge 3 can be classified as a data management problem that demands effective tools and information management mechanisms such as Product Lifecycle Management systems (PLM). Finally, challenge 4 requires formal methods to incorporate various types of design information (Geometric Dimensioning and Tolerancing, manufacturing instructions, etc) within the CAD model. Recent Digital Product definition standards such as ASME Y14.41[14] and ISO 16792 [15] and latest advances in the area of Model-Based Enterprise have facilitated this task to some extent since they have formalized how certain product information must be presented in a 3D model.

The long term vision of model-based engineering approaches is to use CAD models as carriers of design knowledge, where all product information is contained within the geometry. However, for this information to be useful, users wishing to use it must be able to understand the reasons behind modeling decisions and the rationale of the design. One of the key benefits of CAD is the ability to reuse models and designs, but those benefits depend in part on discerning design intent and understanding why the model was created in a certain manner [16, 17]. As projects become more complex, teams more distributed, and workers more transient, capturing design knowledge becomes more important because team members will increasingly work with design knowledge created in part or in whole by other professionals.

In the following section, we examine the concept of design intent and how it relates to reusability. Next, we assess the importance of an explicit representation and explore the challenges we face when developing design intent and knowledge management mechanisms. 


\subsection{Design Intent Communication and Design Annotations}

There is no general consensus on the exact definition of design intent, and many researchers on the subject have suggested their own definitions [18-22]. Nevertheless, the definition proposed by Iyer and Mills [17] after an extensive and comprehensive review that identified elements that were common to all interpretations in the domain of 2D CAD has been widely accepted: "Design intent contained in legacy CAD is the insight into the design variables (design objectives, constraints, alternatives, evolution, guidelines, manufacturing instructions and standards) implicit in the structural, semantic and practical relationships between the geometric, material, dimensional and textual entities present in the CAD representation." [17]. In this research, "design intent" or "geometric design intent" will be used indistinctly to express the reasons that motivate a designer to follow specific CAD modeling procedures so that the model behaves predictably as intended when modified.

The importance of design intent and the benefits of an explicit representation are undeniable. Researchers Pena-Mora et al. [23], summarize these advantages in the form of four points:

- Changes in complex projects require certain design decisions to be modified during the development process. When the justifications defined during the initial stages are lost, they need to be recreated, which has a negative impact on project costs and development times. The ability to store, process, and retrieve this information can significantly improve productivity.

- When design intent information is represented explicitly and is easily available for review, the overall quality of the product increases.

- Explicit representation of design intent leads to a more intelligent use of resources and knowledge.

- Efficient communication of design intent is essential for integrating solutions and transferring design knowledge.

For a CAD model to be easily altered, its design intent must be appropriately captured [24] and understood by the person making the changes $[16,25]$. Despite remarkable advances in CAD technology, many models are often remodeled from scratch [26] due in part to the lack of explicit information about design intent in models created by others [5]. To communicate design intent information effectively, decisions need to be made as to how design intent can be captured, represented, and stored. Although some semi-automated capturing tools have been implemented [27-30], many of them based on IBIS [31], capturing design intent cannot be completely automated [32] and requires designers to be actively involved. Since designers are often reluctant to spend additional time adding information to their models [33], tools to support interaction must be easy and intuitive $[34,35]$. The capture, representation, and retrieval of design intent are still open venues for research [17] and still poorly addressed by current industrial tools [6, 27].

In addition, design intent communication is directly related to CAD quality. According to authors Yan \& Han [36], there are three major types of CAD modeling errors: topological and geometric inaccuracies (such as discontinuities, small and void faces, and self-intersection problems), problems with product data exchange processes (i.e. when models are converted to and from different formats), and incorrect user practices with the CAD system (poor design skills or inexperience of designers). In the latter case, two strategies can be applied: provide a better CAD education that focuses on design intent [26], and use annotations with indications to specific modeling questions to help users understand the reasons behind complex modeling decisions.

Annotations are elements of information used to clarify or explain certain aspects of the object that is being annotated. They are usually textual, but can also take the form of images, audio, video, and hyperlinks. Annotations are employed in many different fields. In software development, for instance, annotations are essential tools in software quality and documentation activities [37-39], where they usually materialize as comments that describe what a program (or part of it) does and how it works. In engineering design, annotations have traditionally been used to complement engineering drawings by providing information that is difficult or impossible to represent otherwise, such as manufacturing instructions, tolerances, and surface finishes.

Three-dimensional CAD environments have supported simple annotations for many years, but it was not until the development of digital product definition standards $[14,15]$ and the popularization of the Model-Based engineering paradigm [1,40] (where all processes revolve around 3D models) that the use of annotations gained significant momentum

In terms of design communication, Boujut and Dugdale [6] emphasized the role of 3D annotations in the cognitive synchronization between designers and as interface elements to mediate interactions between designers. There are also indicators that suggest that annotations can reduce the amount of time required to make alterations to CAD models [41, 42]. However, the fact that 3D models are becoming more complex and comprehensive (carriers of product definition information) demands a renovation (or a redefinition) of traditional model-based annotations, a transformation into 
model-based design annotations. In this context, we observed a lack of experimental results in the scientific literature with evidence of positive effects of design annotations on model reusability.

The main contribution of this paper is the empirical evaluation of $3 \mathrm{D}$ annotations in typical design scenarios that require the communication of certain information that is relevant for altering and reusing a CAD model. In the following sections, we present the results of a series of studies where we assess the suitability and effectiveness of annotations and compare CAD user performance, specifically in terms of model quality and correctness, when interacting with regular non-annotated versus models that were annotated with design information.

\subsection{Annotations in CAD Environments}

Model-based engineering (MBE) standards such as ASME Y14.41 and ISO 16792 have formalized the way product information is presented and interpreted in a three dimensional CAD model, which facilitated the adoption of the MBE paradigm by many engineering firms. The MBE paradigm is a modern approach to product development, where 3D CAD models are used as data sources for all engineering activities throughout the product life cycle [43]. In these environments, annotations are linked to geometric elements in the CAD model and properly arranged without interfering with the model with the purpose of specifying manufacturing and life cycle support data within the geometry itself. When a complete definition is provided, additional documents such as $2 \mathrm{D}$ drawings are not needed.

Model-based standards set general guidelines for the logical association of product information to geometry with the purpose of making CAD models more comprehensive and reusable. Two methods of product definition are defined: model-only, and model and drawing in digital format. Also, two terms are described: annotations (dimension, tolerance, note, text, or symbol visible without any manual or external manipulation) and attributes (dimension, tolerance, note, text, or symbol required to complete the product definition or model feature of the product that is not visible but available upon querying the model.)

Although 3D annotations have been supported by CAD systems for many years, most tools were proprietary and software-dependent, which drastically limited its adoption. Information was difficult to share with other systems and the lack of a common set of rules to regulate annotation practices created inconsistencies in formats and procedures, which had a negative impact in production environments [8]. Annotation mechanisms are now available in modern commercial CAD systems via "Product and Manufacturing Information" (PMI) modules. These modules are implementations of MBE standards and support the creation of Geometric Dimensioning and Tolerancing (GD\&T), 3D textual annotations, surface finishes, and other product specifications in 3D CAD models.

Model annotations are typically represented as blocks of text anchored to specific aspects of a CAD model via leader lines and used as pointers to draw the attention to a specific area of the model [34]. They are usually textual notes or comments, but they can also take other forms such as images, hyperlinks, and references to external documents. Based on their storage method, annotations can be classified as internal (in-line) and external (stand-off). In the first case, annotation information is stored internally within the CAD model. External annotations require the use of external repositories, such as XML files or databases, to store annotation information [44].

In general, external annotations provide greater benefits than internal, as they allow a progressive expansion of the metadata without altering the geometric representation of the model. In addition, multiple annotation files can be used with the same model to provide different levels of annotations for different purposes [45, 46]. However, external annotation strategies are difficult to maintain. Internal annotations, on the other hand, are easier to implement and are already supported by most CAD systems. For the experiments presented in this paper, we use the built-in annotation capabilities of DS SolidWorks ${ }^{\circledR}$, a popular parametric solid modeling package.

\section{EXPERIMENTAL DESIGN}

The framework of our study is the application of $3 \mathrm{D}$ annotations to parametric modeling processes. Our goal is to determine whether annotated models provide a significant benefit over non-annotated models when performing tasks that require manipulation of the model's geometry. With this goal in mind, we formulate two research questions:

$\mathrm{Q}_{1}$ : When modifying a parametric model, are annotations an effective tool to communicate specific design intent information so it is easier for designers to select the most appropriate procedure to perform such modifications?

$\mathrm{Q}_{2}$ : When critical design decisions have to be made, are annotations a valuable tool to explicitly express geometric design requirements and considerations about the model?

For $\mathrm{Q}_{1}$, we identified two important aspects: the difficulty of selecting the most appropriate solution (in terms of model flexibility and reusability) to perform a particular modification to the model when multiple options are available, and the clear understanding of the annotation information and the specific aspect of the model that it refers to. For $\mathrm{Q}_{2}$, 
we focused on the quality and correctness of the model, ensuring that all design requirements expressed by the annotations are met.

Based on the previous questions and framed in terms of research hypotheses, we aim at rejecting:

$\mathrm{H}_{0(1)}$ : Annotations do not have a significant impact on communicating design intent information when altering a parametric model.

$\mathrm{H}_{0(2)}$ : When critical design decisions about a model need to be made, annotated models are not more valuable than non-annotated models in terms of communicating design requirements.

\subsection{Experimental Set-Up and Procedure}

We conducted a series of studies with a group of undergraduate engineering students with previous experience in engineering design graphics and parametric solid modeling, particularly using the CAD package SolidWorks ${ }^{\circledR}$. The experiments were conducted in a computer laboratory environment, where participants were equipped with a workstation and the CAD software. We designed two separate experiments: the first one aimed at model alteration activities, and the second focused on design tasks. For both experiments, participants were randomly divided into two groups. One group served as the control group (participants used non-annotated models to complete the tasks) and the other served as the experimental group (participants used models that were previously annotated by a member of the research team). The annotations we are focusing on are not necessarily design rationale annotations (related to engineering design decisions) but design intent annotations (related to geometric modeling decisions, i.e. why the geometric CAD model was created in a certain way), which are particularly relevant in parametric modeling environments.

In order not to give a clear advantage to the experimental group, no instructions were given regarding the existence or relevance of the annotations. None of the participants had previous experience with annotation techniques and the model was presented as an industrial part created by a professional designer, without mentioning or announcing annotations. In addition, not all annotations included in the model were relevant to the task. Some were prepared to look like personal comments and reminders. Others were more technical in nature. If the full extent of the task was known, then the designer could be informed before initiating work, and obviously perform better. We tried to minimize the amount of information by not providing annotations that give a clear and expected plan of the changes that participants will be called to make.

\subsubsection{Experiment 1}

Our first experiment was intended to obtain new insights on CAD modeling strategies that will allow us to answer $\mathrm{Q}_{1}$ (previously discussed). Our goal was to determine whether annotations are helpful in situations where inadequate assumptions are likely to be made by designers during the modeling process, but can be prevented when design information is explicitly available.

Two activities, each involving a series of alterations to an existing parametric CAD model, were designed for this experiment. The activities were presented in a classic test format, using an online testing tool. Each alteration in the sequence was stated as a separate question, requiring participants to submit their modified CAD model for each question. In order to accurately analyze the modeling process followed by the participants for the entire sequence, only one question was displayed at a time and backtracking was intentionally disabled, so participants were forced to submit a $\mathrm{CAD}$ model for every question before moving to the next, and were not allowed to change the answer to a question that was previously submitted.

\section{Activity 1}

A total of 104 volunteers, randomly divided into two groups of 52, participated in this activity. Participants in both groups received the same model, but a set of ten annotations was added to the model given to the experimental group. Only one of those annotations, indicating not to assume symmetrical arms, is relevant to the task. This annotation is used to communicate that new geometry should not be built as dependant of a mirrored feature, as the original geometry (the arm, in our case) may need to be changed. In this scenario, the annotation provides negative knowledge, as defined by [26], and serves as a warning that is based on the original designer's understanding of the model and previous experiences.

The CAD model provided to the participants is shown in Figure 1. Some information has been intentionally omitted for clarity. 

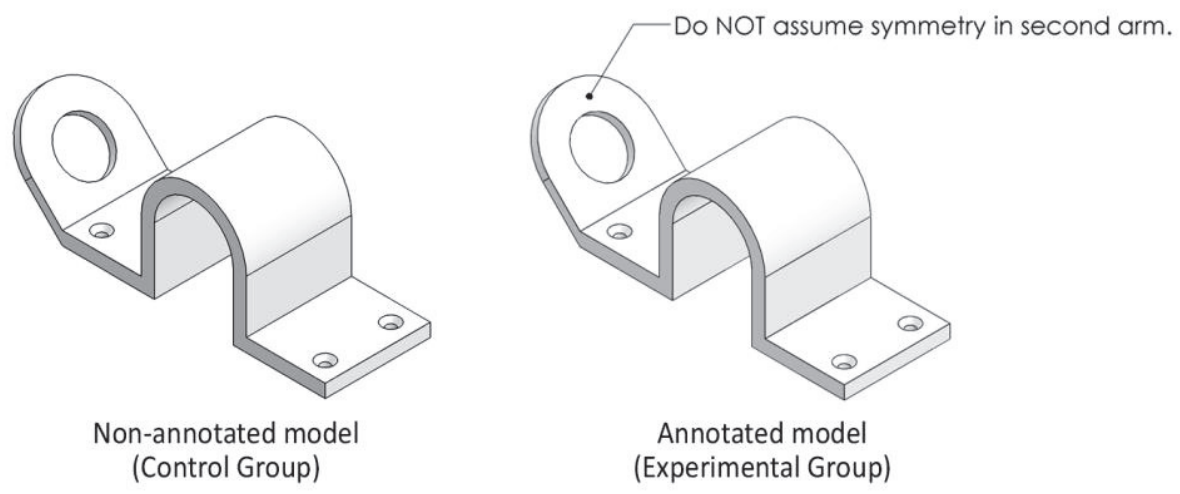

Figure 1. Model provided to participants for Activity 1. In the case of the experimental group (right), dimensions and annotations that are not relevant to the design problem have been intentionally omitted for clarity

The basic steps involved in creating the initial model are represented in Figure 2. Each step is the result of applying a modeling operation using the CAD package.

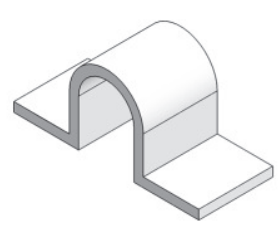

1. Base feature

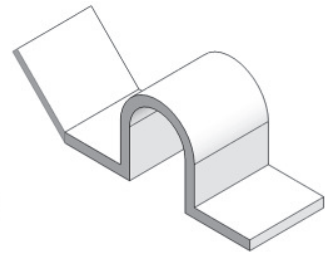

2. Extrude arm

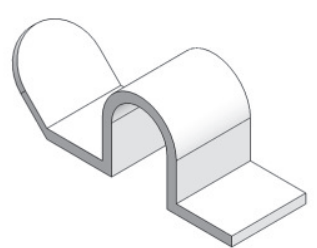

3. Round corners

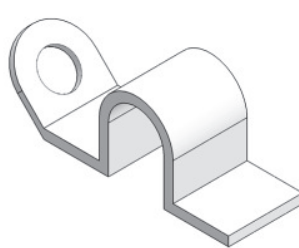

4. Cut hole on arm

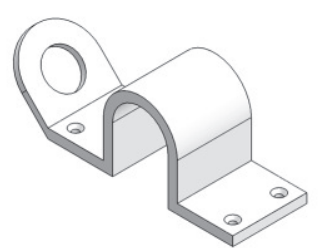

5. Cut machined holes on base

Figure 2. Creation process of original model for activity 1

The sequence of modifications requested is illustrated in Figure 3. Tasks were presented one at a time. Therefore, participants were not allowed to see alteration $i+1$ before completing alteration $i$.

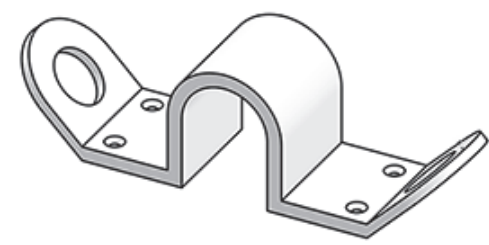

ALTERATION 1

(add second arm)

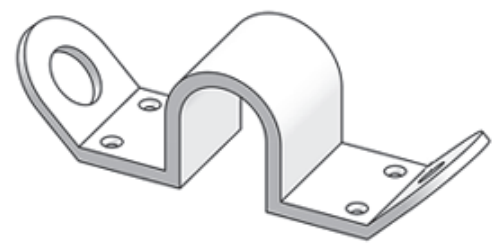

ALTERATION 2

(change hole diameter)

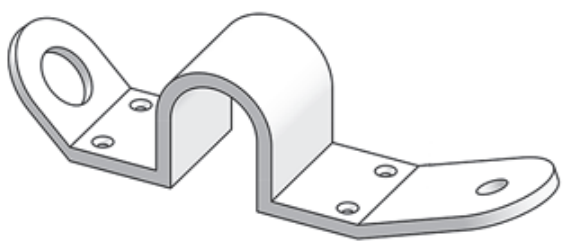

ALTERATION 3

(modify angle and size)

Figure 3. Sequence of alterations requested for activity 1

The first modification task involves the addition of a second arm to the model, identical to the existing arm in both shape and size. There are, at least, two distinctive approaches that can be followed: using symmetry tools to mirror all features in the existing arm, or creating the second arm from scratch by defining a new sketch, extruding it, and cutting a new hole, i.e. repeating the process used to create the original arm (see Figure 4).

Intuitively, the first approach seems easier and faster than the second, as it requires fewer steps and no features need to be modeled from scratch. The result is an efficient model as long as all features in both arms remain symmetrical in new variations of the model. In other words, when selecting this approach, the designer is assuming that both arms are likely to remain symmetrical in future models, which is reasonable if "Alteration 1" is the only piece of information she possesses. Performing the first alteration to the model by creating the second arm from scratch may not seem like an efficient strategy, as it does require a number of additional steps. However, each arm can be controlled and edited separately without affecting the other, should only one arm needed to be modified (which is precisely what happens in upcoming alterations). 


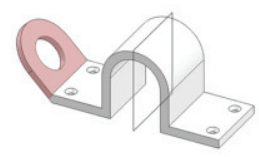

1. Select features to mirror (extrusion, fillet, and cut) and select mirror plane

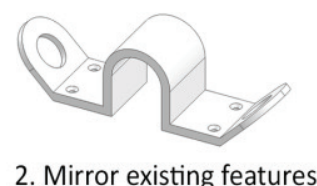

2. Mirror existing features

\section{APPROACH 2: MODEL NEW FEATURES}

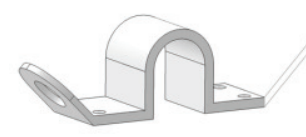

1. Create new sketch

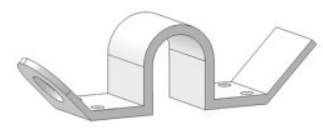

2. Extrude sketch

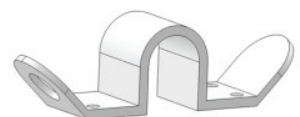

3. Fillet edges

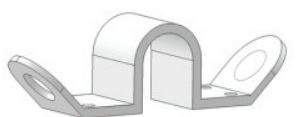

4. Create new sketch

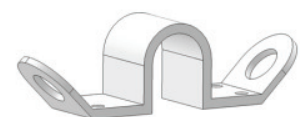

5. Cut hole

Figure 4. Basic approaches for "Alteration 1"

By analyzing the entire sequence, it seems clear that mirroring the existing arm (approach 1) might not be the best approach, since both the angle and the length of the new arm will be modified in Alteration 3 . In fact, if symmetry is assumed, alteration 3 will require the suppression or deletion of all mirrored features and the creation of the new arm from scratch (which is precisely "approach 2"). If we were using a more sophisticated model with a greater number of features that depended on the new arm, performing alteration 3 after mirroring would likely cause major rebuild errors and require a significant amount of time to fix. Since none of this information is available to participants in the control group at the time they have to make a decision about Alteration 1, we hypothesized that:

- If using a non-annotated model, the majority of participants will assume symmetry and use mirror tools to perform Alteration 1.

- If using an annotated model, the majority of participants will create the second arm by modeling the new features from scratch.

We define the modeling approach as the independent categorical variable $\mathrm{X}_{1}$ with values as shown in Table 2:

Table 2. Values of independent variable $\mathrm{X}_{1}$

Description of the modeling approach

\begin{tabular}{|c|lll|}
\cline { 2 - 4 } \multicolumn{1}{c|}{$\mathrm{X}_{1}$} & Alteration 1 & Alteration 2 & Alteration 3 \\
\hline Approach 1 & Mirror arm & Edit previous mirror and create new hole & Delete previous mirror and re-model arm \\
\hline Approach 2* & Model new arm & Edit circle in sketch and update feature & Change dimensions of sketch and update feature \\
\hline Approach 3** & Mirror arm & Delete previous mirror and re-model arm & Change dimensions of sketch and update feature \\
\hline
\end{tabular}

*Most efficient approach, as previously discussed

**When alteration 2 was presented, some participants realized the need to control the two arms separately and decided to re-model the arm from scratch.

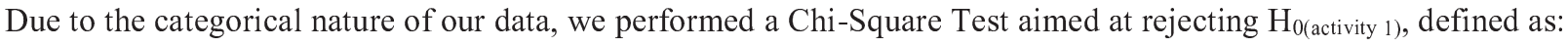

$\mathrm{H}_{0(\text { activity } 1)}$ : There is no statistically significant differences between the experimental and control groups. The annotation does not contribute to the selection of an efficient modeling approach.

$\mathrm{H}_{\mathrm{a}(\text { activity } 1)}$ : There is significant difference between the experimental and control groups. The annotation provided contributes significantly to the selection of an efficient modeling approach.

The results of our study are shown in Table 3.

Table 3. Observed values for activity 1

Modeling approach $\left(\mathrm{X}_{1}\right)$

\begin{tabular}{|l|c|c|c|c|}
\cline { 2 - 5 } \multicolumn{1}{c|}{} & Approach 1 & Approach 2 & Approach 3 & Total \\
\hline Control Group: Non-annotated model & 38 & 10 & 4 & 52 \\
\hline Experimental Group: Annotated model & 11 & 39 & 2 & 52 \\
\hline Total & 49 & 49 & 6 & 104 \\
\hline Chi-square $=32.707$, p-value $<0.001$ & \multicolumn{4}{|l}{} \\
\hline
\end{tabular}


Based on the resulting $p$-value $(p<0.001)$, there is a statistically significant difference between the experimental and control groups. Even with no prior warnings, the design information provided as an annotation contributes to the selection of an efficient modeling approach.

\section{Activity 2}

A total of 77 volunteers (a subset of the group involved in Activity 1), randomly divided into two groups (control group: 38, experimental group: 39) participated in this activity. The tasks involved in this part of the experiment are similar to those proposed in Activity 1. In this case, five alterations were proposed and a number of annotations were inserted into the model provided to the experimental group. Once again, no mention of the existence or relevance of the annotations was given to the experimental group. The CAD model provided to the participants is shown in Figure 5.

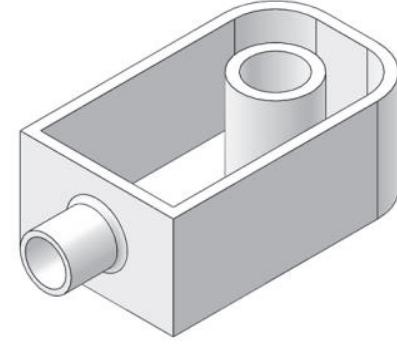

Non-annotated model (Control Group)

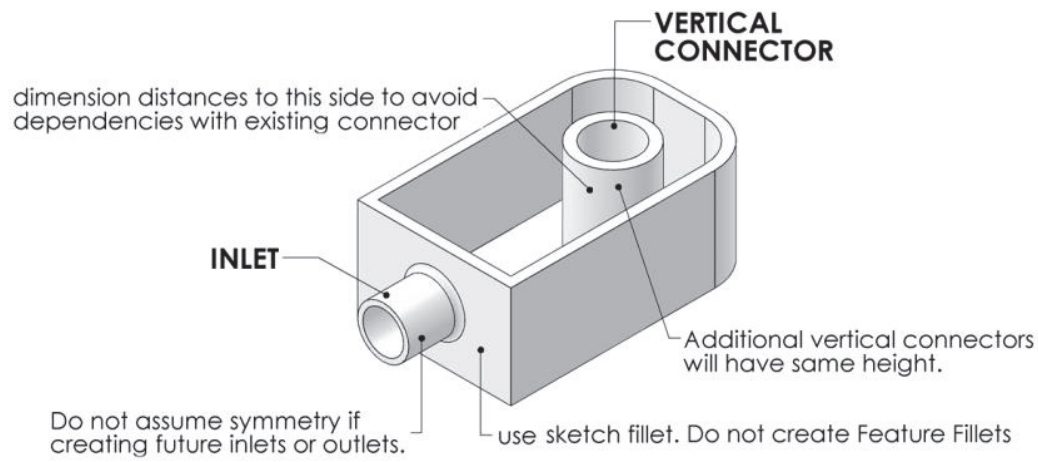

Annotated model

(Experimental Group)

Figure 5. Model provided to participants for activity 2. In the case of the experimental group (right), dimensions and annotations that are not relevant to the design problem are intentionally omitted for clarity

The basic steps involved in creating the model for this activity are represented in Figure 6. Each step is the result of applying a modeling operation using the CAD package.

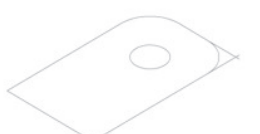

1. Initial sketch

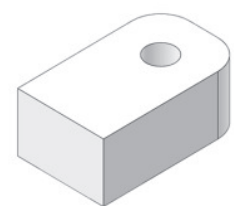

2. Base feature

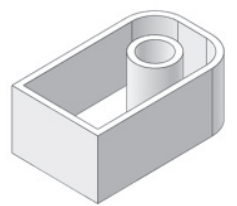

3. Shell top surface

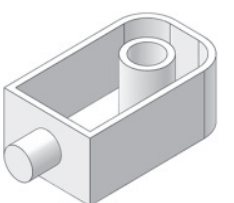

4. Extrude inlet

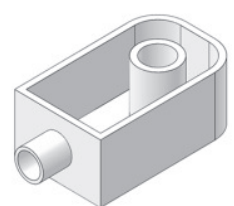

5. Cut hole

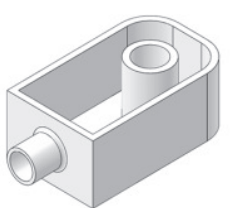

6. Round edge

Figure 6. Creation process of original model for activity 2

The sequence of modifications requested to participants is shown in Figure 7. Similar to the previous activity, tasks were presented one at a time, i.e. participants were not allowed to see alteration $i+1$ until they completed alteration $i$.

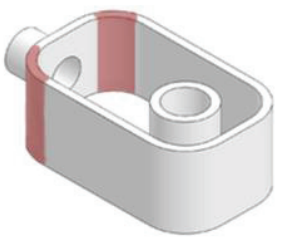

ALTERATION 1 (fillet edges)

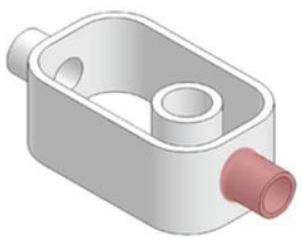

ALTERATION 2 (create outlet)
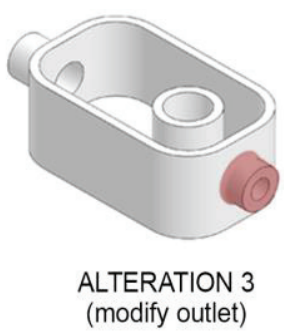

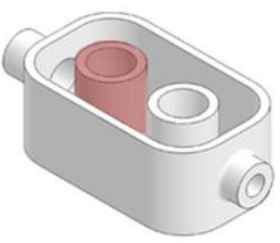

ALTERATION 4 (create new connector)

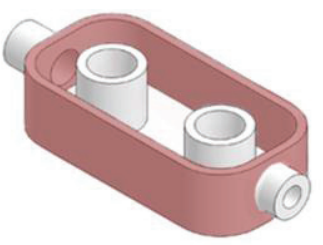

ALTERATION 5 (modify width and height)

Figure 7. Sequence of alterations requested for activity 2 
Based on the influence on later modifications and on the overall model behavior, the sequence of alterations described in Figure 7 can be divided into three tasks:

- Task A: Alteration 1 (the approach taken to perform this alteration does not affect any of the subsequent steps)

- Task B: Alterations 2 and 3 (the approach taken to perform alteration 2 affects alteration 3, but not others)

- Task C: Alterations 4 and 5 (the approach taken to perform alteration 4 affects alteration 5, but not others)

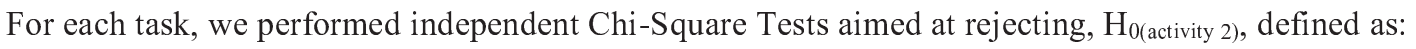

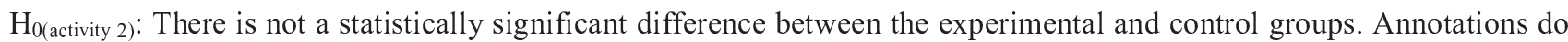
not contribute to the selection of a correct modeling approach.

$\mathrm{H}_{\mathrm{a}(\text { activity } 2)}$ : There is substantial difference between the experimental and control groups. Annotations contribute significantly to the selection of a correct modeling approach.

\section{ACTIVITY 2 - TASK A: FILLET CREATION}

The creation of fillets in 3D models can be performed either at sketch level or feature level. Both methods can produce models that are identical in appearance (see Figure 8). Sketch level fillets involve rounding off the corner at the intersection of two lines in a two-dimensional sketch, whereas feature level fillets create a rounded face on the part based on the selected edge. In general, feature fillets are preferred over sketch fillets, as feature fillets can be edited, deleted, or suppressed independently from the original sketch, they allow more flexibility and control over the model's corners, and they support variable radii, among other advantages. Furthermore, because other features can affect fillets, fillets are usually added toward the end of the modeling process.

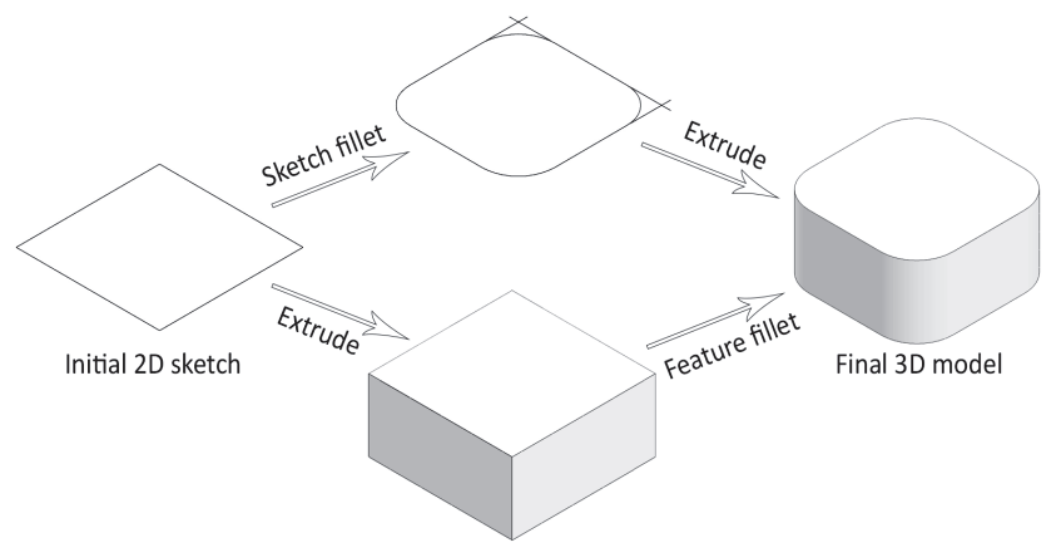

Figure 8. Two approaches to fillet creation

In our example, however, creating the fillets requested in Alteration 1 at feature level produces an incorrect model. The problem is caused by the geometry of the inlet located on the external face and the shell command used to hollow out the top surface. Selecting the inner and outer vertical edges of the model to create feature fillets causes the filleted arcs to lose concentricity, creating a thicker wall on that side of the model (see Figure 9). In this situation, feature fillets are not only inefficient, but also incorrect. Therefore, sketch fillets must be used. The concentricity constraint at sketch level ensures constant wall thickness at 3D level. We hypothesized that:

- If using a non-annotated model, the majority of participants will create the requested fillets at feature level, based on the preconceived advantages of this method without realizing the problematic effects in this specific situation.

- If using an annotated model, the majority of participants will create sketch fillets, preventing the problem shown in Figure 9. 

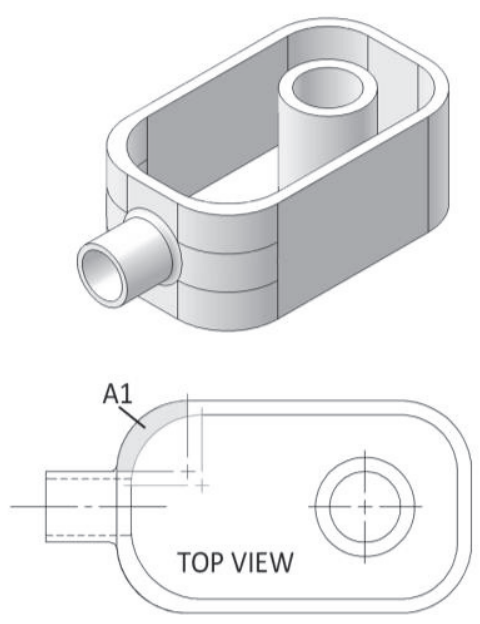

FEATURE FILLETS

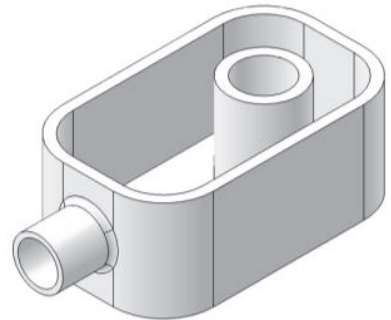

$\mathrm{A} 1>\mathrm{A} 2$

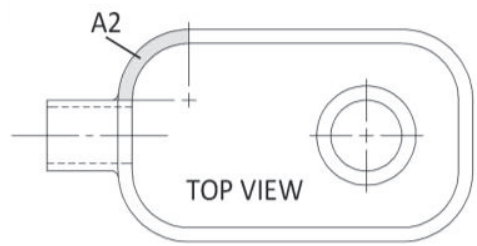

SKETCH FILLETS

Figure 9. Feature fillets vs. Sketch fillets

The results of our study are shown in Table 4.

Table 4. Observed values for activity 2 (fillet creation)

Modeling approaches

\begin{tabular}{|l|c|c|c|}
\cline { 2 - 4 } \multicolumn{1}{c|}{} & Sketch fillet & Feature fillet & Total \\
\hline Control Group: Non-annotated model & 5 & 33 & 38 \\
\hline Experimental Group: Annotated model & 28 & 11 & 39 \\
\hline Total & 33 & 44 & 77 \\
\hline Chi-square $=27.022$, p-value $<0.001$ & \multicolumn{3}{c}{} \\
\hline
\end{tabular}

Based on the resulting $p$-value $(p<0.001)$, there is a statistically significant difference between the experimental and control groups. Even with no prior warnings, the design information provided as an annotation contributes to the selection of an efficient modeling approach.

\section{ACTIVITY 2 - TASK B: OUTLET}

The tasks in this group are similar to the ones designed for activity 1 . First, the addition of an outlet, identical to the existing inlet in both shape and size, is requested. There are two approaches that can be followed: using symmetry tools to mirror all features in the inlet, or creating the outlet from scratch by defining a new sketch, extruding it, and cutting a new hole, i.e. repeating the process used to create the original inlet.

Once again, the first approach seems a better option, as long as all features in the outlet remain symmetrical to the inlet in new variations of the model. As we observed in activity 1, this is a reasonable assumption when the designer only possesses a limited piece of information. Modeling the outlet from scratch, however, allows us to control the outlet independently from the inlet, which is helpful when future modifications such as "Alteration 3" need to be performed. The value of modeling the outlet from scratch instead of mirroring the existing inlet becomes more obvious when more drastic modifications are necessary, such as changing the location of the outlet, creating multiple outlets on the same surface, or significantly redesigning its shape. For this task, we hypothesized that:

- If using a non-annotated model, the majority of participants will assume symmetry and use mirror tools to create the outlet features.

- If using a model that was previously annotated with relevant design information, the majority of participants will model the outlet from scratch.

We define the modeling approach as the independent categorical variable $\mathrm{X}_{2}$ with values as shown in Table 5 . The results of our study are shown in Table 6 . 
Table 5. Values of independent variable $\mathrm{X}_{2}$

\begin{tabular}{|l|ll|}
\cline { 2 - 3 } \multicolumn{1}{c|}{} & \multicolumn{2}{c|}{ Description of the modeling approach } \\
\cline { 2 - 3 } \multicolumn{1}{c|}{} & Alteration 1 & Alteration 2 \\
\hline Approach 1 & Mirror inlet to create outlet & Delete mirror and re-model outlet with new dimensions \\
\hline Approach 2* & Model outlet from scratch & Edit outlet diameter and length \\
\hline Approach 3** & Mirror inlet to create outlet & New sketch on mirrored feature and cut to reduce outlet length \\
\hline
\end{tabular}

*Most efficient approach, as previously discussed

**Approach 3 is an inefficient approach taken by some participants. In this case, updating the dimensions of the outlet requires making changes to three or more different features.

Table 6. Observed values for activity 2 (outlet creation)

Modeling approaches $\left(\mathrm{X}_{2}\right)$

\begin{tabular}{|l|c|c|c|c|}
\cline { 2 - 5 } \multicolumn{1}{c|}{} & Approach 1 & Approach 2 & Approach 3 & Total \\
\hline Control Group: Non-annotated model & 21 & 15 & 2 & 38 \\
\hline Experimental Group: Annotated model & 6 & 30 & 3 & 39 \\
\hline Total & 27 & 45 & 5 & 77 \\
\hline Chi-square=13.33, p-value $<0.001$ & \multicolumn{4}{|l}{} \\
\hline
\end{tabular}

Based on the resulting $p$-value $(p<0.001)$, there is a statistically significant difference between the experimental and control groups. Even with no prior warnings, the design information provided as an annotation contributes substantially to the selection of an efficient modeling approach.

\section{ACTIVITY 2 - TASK C: VERTICAL CONNECTOR AND OVERALL SIZE}

For the last two alterations, three major approaches can be taken. Two of these can be further divided based on the selection of dimensional constraints (see Figure 10). Because the overall width and height of the part will change in alteration 5, the goal is to optimize the model, so it is flexible enough to automatically adjust to the changes. In this case, since both vertical connectors will remain identical in size and symmetrical, approaches $1 \mathrm{~b}$ and 3 are the most efficient modeling strategies. Based on the information provided in the annotations for this task, we hypothesized that:

- If using a non-annotated model, the majority of participants will not select an efficient modeling strategy to create the new vertical connector.

- If using an annotated model, the majority of participants will select an efficient modeling strategy (approaches $1 \mathrm{~b}$ or 3 ) to model the new connector.

APPROACH 1: EDIT INITIAL SKETCH

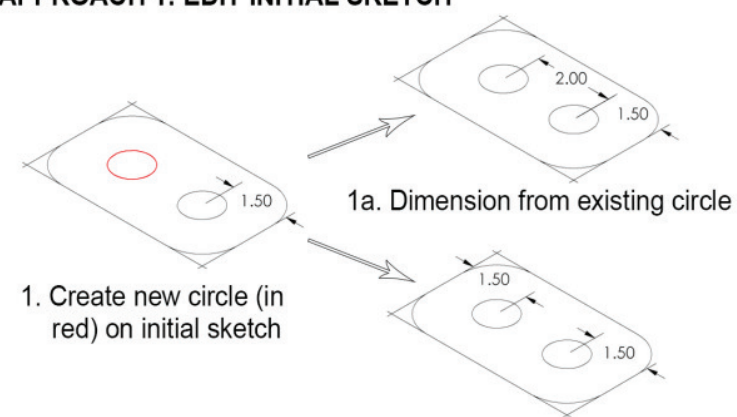

1b. Dimension from side
APPROACH 2: NEW SKETCH

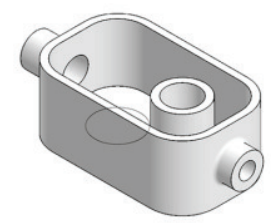

2. Create new sketch on surface

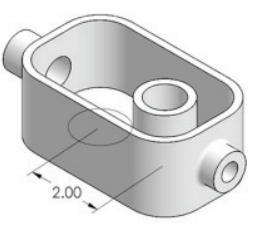

2a. Dimension from existing connector

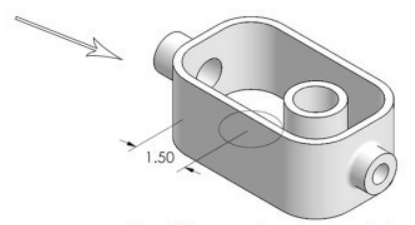

2b. Dimension from side

\section{APPROACH 3: MIRROR CONNECTOR}

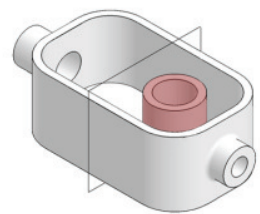

3. Mirror existing connector about plane

Figure 10. Modeling approaches to new vertical connector 
The results of our study are shown in Table 7.

Table 7. Observed values for activity 2 (new connector)

Modeling approaches

\begin{tabular}{|l|c|c|c|c|c|c|}
\cline { 2 - 7 } \multicolumn{1}{c|}{} & Approach 1a & Approach 1b & Approach 2a & Approach 2b & Approach 3 & Total \\
\hline Control Group: Non-annotated model & 5 & 2 & 17 & 13 & 1 & 38 \\
\hline Experimental Group: Annotated model & 3 & 17 & 5 & 11 & 3 & 39 \\
\hline Total & 8 & 19 & 22 & 24 & 4 & 77 \\
\hline Chi-square=20.045, p-value $<0.001$ & \multicolumn{5}{|l}{} \\
\hline
\end{tabular}

Based on the resulting $p$-value $(\mathrm{p}<0.001)$, there is a statistically significant difference between the experimental and control groups. Even with no prior warnings, the design information provided as an annotation contributes substantially to the selection of an efficient modeling approach.

\subsubsection{Experiment 2}

The goal of our second experiment is to determine whether annotations are a valuable tool to communicate requirements, constraints, and modeling considerations in situations where design decisions need to be made. Rather than making specific changes to an existing model, in this experiment participants are asked to find a solution to a design problem that can be solved by taking different approaches. A total of 104 participants (52 in the control group in 52 in the experimental group) were part of this study. The models provided to participants are shown in Figure 11.
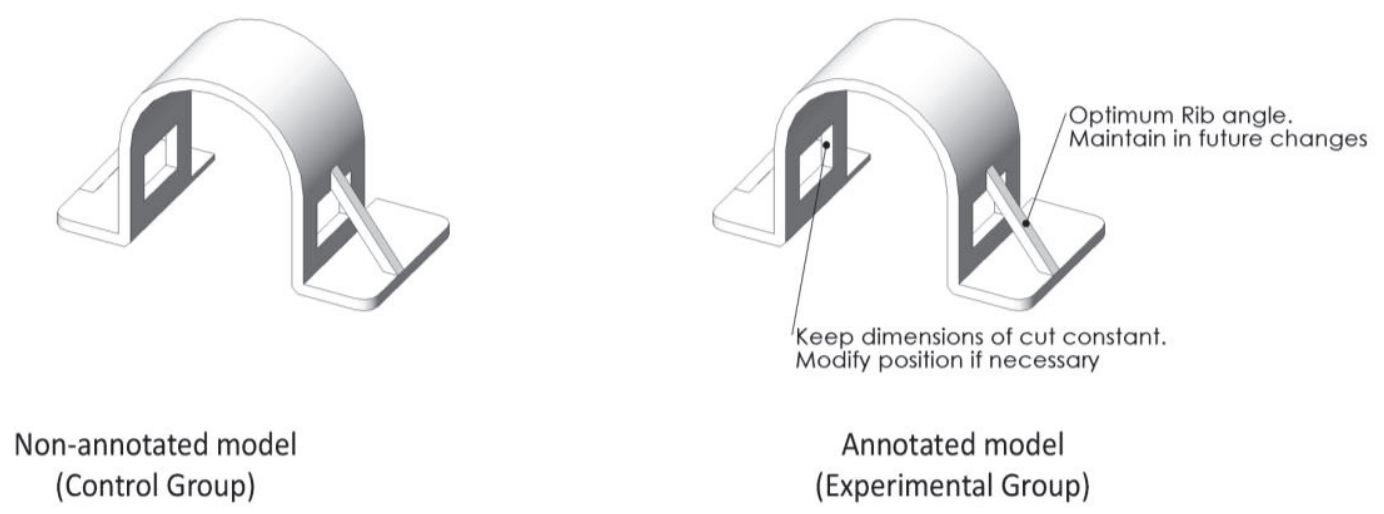

Figure 11. Model provided to participants for experiment 2

As a first step, participants were asked to change the dimensions of the side ribs from $4 \mathrm{~mm}$ to $5 \mathrm{~mm}$ (see Figure 12). This alteration is intentionally prepared to cause an unwanted effect in the model (see Figure 13). Participants were then challenged to find a solution to this undesired geometry.
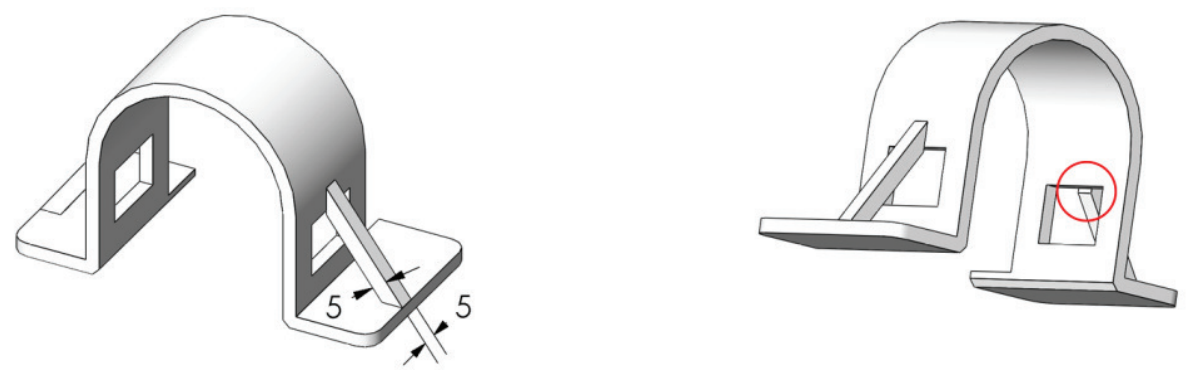

Figure 12. First alteration (left) causes unwanted effect (shown in circle)

We identified three major approaches to this problem: increasing the angle of the ribs, reducing the dimensions of the square cuts on both sides of the part, or changing the position of the side cuts by moving them closer to the bottom (see Figure 13). 


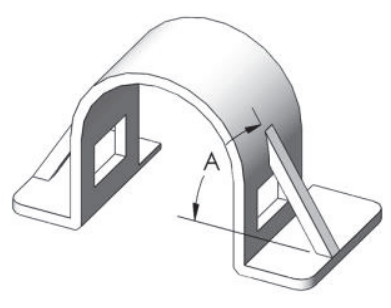

Solution 1: Increase rib angle

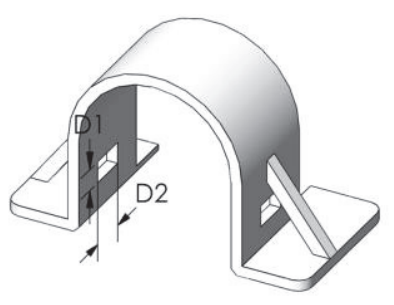

Solution 2: Change side cut dimensions

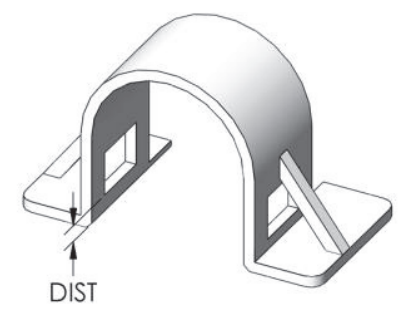

Solution 3. Move side cut down

Figure 13. Possible solutions to design problem

The information included in the annotated model contains remarks that the original designer of the part considered valuable. This knowledge explicitly suggests maintaining the current rib angle and the dimensions of the side cut. These restrictions and design requirements will certainly determine how the model must be altered and what design conditions need to be met at all times. Therefore, according to the annotations, modifying the position of the side cuts (solution 3 ) is the most effective approach.

Based on this information, we performed a Chi-Square Test aimed at rejecting, with a level of significance $\alpha=0.05$, the null hypothesis H0(activity 2 ), defined as:

- $\quad \mathrm{H}_{0(2)}$ : When critical design decisions about a model need to be made, annotated models are not more valuable than non-annotated models in terms of communicating design requirements.

- $\mathrm{H}_{\mathrm{a}(2)}$ : When critical design decisions about a model need to be made, annotated models are more valuable than non-annotated models in terms of communicating design requirements.

We define the modeling approach as the independent categorical variable $\mathrm{X}_{3}$ with values as shown in Table 8. The results of our study are shown in Table 9.

Table 8. Values of independent variable $\mathrm{X}_{3}$

$\mathrm{X}_{3} \quad$ Description of the modeling approach

\begin{tabular}{|c|c|}
\hline Solution 1 & Increase rib angle \\
\hline Solution 2 & Change cut dimensions \\
\hline Solution 3 & Move side cut down \\
\hline Solution $4 *$ & Other approaches \\
\hline
\end{tabular}

*Other approaches include incorrect approaches, such as adding new geometry to the cut as a new feature (inefficient), or trimming the size of the rib (incorrect, since the rib must remain at $5 \mathrm{~mm} \times 5 \mathrm{~mm}$ )

Table 9. Observed values for experiment 2

Modeling approaches $\left(\mathrm{X}_{3}\right)$

\begin{tabular}{|l|c|c|c|c|c|}
\cline { 2 - 6 } \multicolumn{1}{c|}{} & Solution 1 & Solution 2 & Solution 3 & Solution 4 & Total \\
\hline Control Group: Non-annotated model & 7 & 32 & 9 & 4 & 52 \\
\hline Experimental Group: Annotated model & 9 & 12 & 25 & 6 & 52 \\
\hline Total & 15 & 44 & 34 & 10 & 104 \\
\hline Chi-square=17.27, p-value $<0.001$ & \multicolumn{4}{|c|}{} \\
\hline
\end{tabular}

Based on the resulting $\mathrm{p}$-value $(\mathrm{p}<0.001)$, there is a statistically significant difference between the experimental and control groups. When critical design decisions about a model need to be made, annotated models are more valuable than non-annotated models in terms of communicating design requirements.

\section{Conclusions and Future Work}

As organizations migrate to the Model-Based Engineering paradigm and other concurrent methodologies, more importance is put on digital models to drive engineering activities and greater amounts of design data is generated and shared. Reusing model information throughout the lifecycle of a product and sharing it with other 
members of the team, typically with diverse backgrounds, requires quality models, an efficient communication infrastructure, and new methods to support, capture, manage, and present information.

The role of 3D annotations as mechanisms to incorporate information within CAD models has been found successful. However, their ability to communicate design intent explicitly and facilitate reusability is substantially more challenging, although there are reasons to believe they can serve this purpose effectively as long as proper procedures are established.

In this study, we laid the foundation for understanding the potential of annotated models in the context of reusability and their limitations in terms of communication. We evaluated user's performance in simulated scenarios that involved the alteration of annotated CAD models. Our objective was to find evidence of better responses and model quality when annotations were used to communicate design intent.

Although we consider this study still in its early stages, the preliminary results suggest that users perform better in terms of modeling quality and reusability when design intent information is explicitly available as annotations. We are preparing a more comprehensive study with a larger and wider variety of models, representing a more diverse range of design scenarios, and a larger and more experienced sample of CAD users.

Our research team tried to minimize the number of potential hints that could unintentionally be given to users regarding subsequent modeling steps by providing a large number of annotations (most of them irrelevant to the task) in each model and not giving any specific instructions about the existence or significance of the annotations. In the near future, we plan to conduct a similar study where participants will be shown the initial and final versions of a more complex CAD model and asked to perform a longer sequence of alterations. We will evaluate whether annotations have a significant impact on design intent communication (so users perform better), even when the geometry modifications are exposed at the beginning of the exercise.

In addition, it will be interesting to compare user's performance when design intent is available as annotations versus when it is provided as a traditional document or included in an external repository. Further work is also required to evaluate user acceptance, motivation, and incentives regarding the annotation of models. As a long term goal, we envision the development of automated mechanisms that take full advantage of comprehensive CAD models. For example, different aspects of the annotation such as language, writing style, and content can be analyzed and used to define CAD quality metrics and sets of best modeling practices.

\section{References}

1. Frechette SP, 2011. Model Based Enterprise for Manufacturing. 44th CIRP International Conference on Manufacturing Systems. Madison, WI.

2. Toussaint J, Cheng K, 2002. Design Agility and Manufacturing Responsiveness on the Web. Integrated Manufacturing Systems 13(5), pp. 328-339.

3. Ullman, DG, 2010. The Mechanical Design Process, 4th ed. Mc Graw Hill, Boston.

4. Bodein Y, Rose B, Caillaud E, 2014. Explicit reference modeling methodology in parametric CAD system. Computers in Industry 65(1), pp. 136-147.

5. Salehi V, McMahon C, 2009. Action Research into the Use of Parametric Associative CAD Systems in an Industrial Context. Proceedings of the 17th International Conference on Engineering Design, ICED09, vol. 5, pp. 133-144.

6. Boujut JF, Dugdale J, 2006. Design of a 3D Annotation Tool for Supporting Evaluation Activities in Engineering Design. Cooperative Systems Design, COOP vol. 6, pp.1-8.

7. Davies D, McMahon CA, 2006. Multiple Viewpoint Design Modelling through Semantic Markup. Proceedings of ASME 2006 International Design Engineering Technical Conferences and Computers and Information in Engineering Conference, Philadelphia, Pennsylvania, USA, vol. 3, pp. 561-571.

8. Dorribo-Camba J, Alducin-Quintero G, Perona P, Contero M, 2013. Enhancing Model Reuse through 3D Annotations: A Theoretical Proposal for an Annotation-Centered Design Intent and Design Rationale Communication. Proceedings of the ASME International Mechanical Engineering Congress \& Exposition, paper no. IMECE2013-64595, San Diego, CA, USA

9. Chang KH, Silva J, Bryant I, 1999. Concurrent Design and Manufacturing for Mechanical Systems. Concurrent Engineering 7, pp. 290-308.

10. Jackson C, Buxton M, 2007. The Design Reuse Benchmark Report: Seizing the Opportunity to Shorten Product Development. Aberdeen Group, Boston.

11. Lang SYT, Dickinson J, Buchal RO, 2002. Cognitive Factors in Distributed Design. Computers in Industry 48, pp. 8998. 
12. Iyer N, Jayanti S, Lou K, Kalyanaraman Y, Ramani K, 2005. Shape-based Searching for Product Lifecycle Applications. Computer-Aided Design 37(13), pp. 1435-1446.

13. Bodein, Y, Rose B, Caillaud E, 2013. A Roadmap for Parametric CAD Efficiency in the Automotive Industry. Computer-Aided Design 45(10), pp. 1198-1214.

14. ASME, 2012. ASME Y14.41-2012 Digital Product Definition Data Practices. The American Society of Mechanical Engineers, New York.

15. ISO, 2006. ISO 16792:2006 Technical Product Documentation - Digital Product Definition Data Practices. Organisation Internationale de Normalisation, Genève, Suisse.

16. Regli,WC, Hu X, Atwood M, Sun W, 2000. A survey of design rationale systems: Approaches, representation, capture and retrieval. Engineering with Computers 16, pp. 209-235.

17. Iyer GR, Mills JJ, 2006. Design Intent in 2D CAD: Definition and Survey. Computer-Aided Design and Applications 3(1-4), pp. 259-267.

18. Conklin EJ, Yakemovic KC, 1991. A Process Oriented Approach to Design Rationale. Human-Computer Interaction 6, pp 357-391.

19. Nielsen E, Dixon J, Zinsmeister G, 1991. Capturing and Using Designer Intent in a Design-With-Features System. Proceedings Design Theory and Methodology, DE 31, pp. 95-102.

20. Lee J, Lai K, 1991. What's in design rationale. Human-Computer Interaction 6(3-4), pp. 251-280.

21. Horvath L, Rudas IJ, 2003. Modeling Behavior of Engineering Objects Using Design Intent Model. Industrial Electronics Society. IECON'03. 29th Annual Conference of the IEEE, 1, pp. 872-876.

22. Brissaud D, Garro O, Poveda O, 2003. Design Process Rationale Capture and Support by Abstraction of Criteria. Research in Engineering Design 14, pp 162-172.

23. Pena-Mora F, Sriram D, Logcher R, 1993. SHARED-DRIMS: SHARED Design Recommendation-Intent Management System. Enabling Technologies: Infrastructure for Collaborative Enterprises, Proceedings, Second Workshop, pp 213 221.

24. Rodriguez J, Ridge J, Dickinson A, Whitwam R, 1998. CAD Training Using Interactive Computer Sessions. Proceedings of the 1998 ASEE Annual Conference and Exposition. Seattle, WA, USA.

25. Karsenty L, 1996. An Empirical Evaluation of Design Rationale Documents. Proceedings of the SIGCHI Conference on Human Factors in Computing Systems. Vancouver, British Columbia, Canada.

26. Mandorli F, Otto HE, 2013. Negative Knowledge and a Novel Approach to Support MCAD Education. ComputerAided Design and Applications 10(6), pp.1007-1020.

27. Bracewell RH, Wallace KM, 2003. A Tool for Capturing Design Rationale. 14th International Conference on Engineering Design, Design Society, Stockholm, Sweden.

28. Ganeshan R, Garrett J, Finger S, 1994. A Framework for Representing Design Intent. Design Studies 15(1), pp. $59-84$.

29. Quereshi S, Shah J, Urban SD, Harter E, Parazzoli C, Bluhm T, 1997. Integration Model to Support Archival of Design History in Databases. ASME Design Engineering Technical Conferences and Computers in Engineering Conference.

30. Myers K, Zumel N, Garcia P, 2000. Acquiring Design Rationale Automatically. Artificial Intelligence for Engineering Design, Analysis and Manufacturing 14(2), pp. 115-135.

31. Kunz W, Rittel HWJ, 1970. Issues as Elements of Information Systems. Working paper 131. Center for Planning and Development Research, Berkeley.

32. Shum SJB, Selvin AM, Sierhuis M, Conklin J, Haley CB, Nuseibeh B, 2006. Hypermedia Support for ArgumentationBased Rationale: 15 Years on from Gibis and Qoc. Rationale Management in Software Engineering, pp. 111-132.

33. Szykman S, Sriram R, Regli W, 2001. The Role of Knowledge in Next-Generation Product Development Systems. Journal of Computing and Information Science in Engineering 1(1), pp. 3-11.

34. Ding L, Ball A, Patel M, Matthews J, Mullineux G, 2009. Strategies for the Collaborative Use of CAD Product Models. 17th International Conference on Engineering Design, vol. 8, pp. 123-134.

35. Chandrasegaran SK, Ramani K, Sriram RD, Horváth I, Bernard A, Harik RF, Gao W, 2013. The Evolution, Challenges, and Future of Knowledge Representation in Product Design Systems. Computer-Aided Design 45(2), pp. 204-228.

36. Yang J, Han S, 2006. Repairing CAD model errors based on the design history. Computer Aided Design 38(6), pp. 627640 .

37. Kajko-Mattsson M, 2001. The State of Documentation Practice within Corrective Maintenance. Proceedings of the IEEE International Conference on Software Maintenance (ICSM'01).

38. Van De Vanter ML, 2002. The documentary structure of source code. Information and Software Technology 44, pp. 767-782.

39. Glass RL, 2003. Facts and Fallacies of Software Engineering: Addison-Wesley 
40. Whittenburg M, 2012. Model-Based Enterprise: An Innovative Technology-Enabled Contract Management Approach. Journal of Contract Management 10, pp. 103-112.

41. Alducin-Quintero G, Contero M, Martín-Gutiérrez J, Guerra-Zubiaga DA, Johnson MD, 2011. Productivity Improvement by Using Social-Annotations About Design Intent in CAD Modeling Process. Online Communities and Social Computing. Springer Berlin Heidelberg. Lecture Notes in Computer Science 6778, pp.153-161.

42. Alducin-Quintero G, Rojo A, Plata F, Hernández A, Contero M, 2012. 3D Model Annotation as a Tool for Improving Design Intent Communication: A Case Study on Its Impact in the Engineering Change Process. Proceeding of the ASME International Design Engineering Technical Conferences and Computers and Information in Engineering Conference, Vol.2, pp. 349-356, Chicago, IL.

43. Lubell J, Chen K, Horst J, Frechette S, Huang P, 2012. Model Based Enterprise/Technical Data Package Summit Report. NIST Technical Note 1753.

44. Ding L, Liu S, 2010. Markup in Engineering Design: A Discourse. Future Internet, 2, pp. 74-95.

45. Patel M, Ball A, Ding L, 2008. Curation and Preservation of CAD Engineering Models in Product Lifecycle Management. Conference on Virtual Systems and Multimedia Dedicated to Digital Heritage (VSMM'08), University of Bath, pp. 59-66.

46. Li C, McMahon C, Newnes L, 2009. Annotation in Product Lifecycle Management: A Review of Approaches. Proceedings of the ASME International Design Engineering Technical Conferences and Computers and Information in Engineering Conference, DETC2009. Vol. 2. New York: ASME, pp. 797-806. 